large integral values may be avoided by preliminary or intermediate treatment of the matrix, the creeping decimal error is ever present and may be met only with otherwise unnecessary decimals or with iterative methods. ${ }^{3}$

The time consumed by this method and the time by the method of all complete reductions were in the ratio of 1 to 3 when using the same equipment in both cases and with the same number handicap, by the same operators.

Gabriel G. Bejarano

BRUCE R. ROSENBLATt

California Research Corporation

Richmond, California

1 A. C. Artken, Determinants and Matrices. Edinburgh, 5th ed., 1948.

2 P. S. DwYer, Linear Computations. New York, 1951.

3 H. Hotelling, "Some new methods in matrix calculation," Annals Math. Stat., v. 14, 1943 , p. 1-34.

"P. D. CROUT, "A short method for evaluating determinants and solving systems of linear equations with real or complex coefficients," AIEE, Trans., v. 60, 1941, p. 1235-1241.

${ }_{5}^{5} \mathrm{G}$. W. PETRIE III, "Matrix inversion and solution of simultaneous linear algebraic equations with the IBM type 604 Electronic Calculating Punch," IBM Computation Seminar, August 1951, Proc., p. 105-111.

'J. Chancellor, J. W. Sheldon, \& G. L. Tatum, "The solution of simultaneous linear equations using the IBM Card-Programmed Electronic Calculator," IBM Industrial Computation Seminar, September 1950, Proc., p. 57-61.

7 W. D. BELL, "Punched card techniques for the solution of simultaneous equations and other matrix operations," IBM Scientific Computation Forum, 1948, Proc., p. 28-31.

${ }^{8} \mathrm{~J}$. vON NEUMANN \& H. H. GoldsTINE, "Numerical inverting of matrices of high order," Amer. Math. Soc., Bull., v. 53, 1947, p. 1021-1099.

A. OPLER, "Monte Carlo matrix calculation with punched card machines," MTAC, v. 5,1951 , p. $115-120$.

$10 \mathrm{~J}$. SHERMAN, "Computations of inverse matrices by means of IBM machines," IBM Technical Newsletter, no. 3, 1951, p. 22-25.

$11 \mathrm{~J}$. LowE, "Solution of simultaneous linear algebraic equations using the IBM type 604 Electronic Calculating Punch," IBM Computation Seminar, Dec. 1949, Proc., p. 54-56.

${ }_{12}$ F. M. VERZUH, "The solution of simultaneous linear equations with the aid of the 602 Calculating Punch," MTAC, v. 3, p. 453-462.

\title{
Error Bounds on Approximate Solutions to Systems of Linear Algebraic Equations
}

1. The Abmatrix. Consider the real matrix $A=\left(a_{i j}\right), i=1(1) m, j=$ $1(1) n$. Define $\alpha(A)$, read "the abmatrix of $A$," to be

$$
\alpha(A)=\left(\left|a_{i j}\right|\right) .
$$

Thus, $\alpha(A)$ is the matrix having each element equal to the absolute value of the corresponding element of $A$. In particular, for a scalar $s$,

$$
\alpha(s)=|s| \text {. }
$$

If $A=\left(a_{i j}\right)$ and $B=\left(b_{i j}\right), i=1(1) m, j=1(1) n$, define

$$
\alpha(A) \leqslant \alpha(B)
$$

to mean

$$
\left|a_{i j}\right| \leqslant\left|b_{i j}\right| \quad \text { for all } i, j \text {. }
$$

Thus, if $\alpha(A) \leqslant \alpha(B)$ and $\alpha(B) \leqslant \alpha(C)$, then $\alpha(A) \leqslant \alpha(C)$. 
From these definitions, it follows that if the operations $A+B$ and $A B$ are permissible, then

$$
\alpha(A+B) \leqslant \alpha(A)+\alpha(B),
$$

and

$$
\alpha(A B) \leqslant \alpha(A) \alpha(B) .
$$

Extensions to and combinations of (1) and (2) are indicated. In particular, if $\boldsymbol{A}$ is a square matrix and $p$ is a positive integer,

$$
\alpha\left(A^{p}\right) \leqslant[\alpha(A)]^{p} .
$$

Subsequently, $[\alpha(A)]^{p}$ will be written $\alpha^{p}(A)$.

This simple notion of the abmatrix is readily applicable to examining the convergence of a class of iterative methods for solving systems of linear algebraic equations and to determining error bounds for approximate solutions of these linear systems; however, in this note, only the latter will be examined. For their application, the use of an approximate inverse is required.

2. The Error in the Approximate Solution. Let the linear system under consideration be

$$
A \xi=\eta,
$$

where $A$ is non-singular and of order $n$. Let $G$ be an approximate inverse of $A$, the approximate solution being

Then,

$$
x=G \eta
$$

$$
\xi=\sum_{h=0}^{p}(I-G A)^{h} x+(I-G A)^{p+1} \xi,
$$

which is simply proven by induction.

Denote now the error in $x$ by $\epsilon=\xi-x$, and let $D=I-G A$. Thus, from (3),

$$
\epsilon=\sum_{h=1}^{p} D^{h} x+D^{p+1} \xi .
$$

Restrict $G$ by the condition that $D^{p} \rightarrow 0$ as $p \rightarrow \infty$. Then,

$$
\epsilon=\sum_{h=1}^{\infty} D^{h} x,
$$

and this expression will be the basis for placing bounds on the error $\epsilon$.

3. Requirements on $D$ and First Uses of the Abmatrix. In Part 2 it was required that $D^{p} \rightarrow 0$ as $p \rightarrow \infty$. By means of the abmatrix, easy sufficient conditions for this convergence can be found.

Let $\gamma=\left(g_{i}\right), i=1(1) n$, be a column vector with $g_{i}>0$. It is always possible to find a scalar $k$, depending on $\gamma$, such that

$$
\alpha(D) \gamma \leqslant k \gamma \text {. }
$$


It then follows that

$$
0 \leqslant \alpha\left(D^{p}\right) \gamma \leqslant \alpha\left(D^{p-1}\right) \alpha(D) \gamma \leqslant k \alpha\left(D^{p-1}\right) \gamma .
$$

Thus, by induction,

$$
0 \leqslant \alpha\left(D^{p}\right) \gamma \leqslant k^{p} \gamma .
$$

Hence, if $k<1, D^{p} \rightarrow 0$ as $p \rightarrow \infty$.

In conclusion, (5) with $k<1$ is a sufficient condition for the desired convergence. Clearly, the same arguments hold for a row vector $\gamma^{\prime}$.

Suppose then that $k<1$ for some specified column vector $\gamma$. Bounds on $\sum_{h=1}^{\infty} D^{h}$ may be found by column-wise examination. Consider $\sum_{h=1}^{\infty} D^{h} e_{j}$ where $e_{j}$ is the $j$ th column of $I$. Then,

Since

$$
\alpha\left[\sum_{h=1}^{\infty} D^{h} e_{j}\right] \leqslant \sum_{h=1}^{\infty} \alpha\left(D^{h} e_{j}\right) .
$$

$$
\alpha(D) e_{j} \leqslant f_{j} \gamma,
$$

where $f_{j}$ is a scalar depending on $\gamma$, it follows that

and for $k<1$,

$$
\alpha\left[\sum_{h=1}^{\infty} D^{h} e_{j}\right] \leqslant \sum_{h=0}^{\infty} f_{j} k^{h} \gamma,
$$

$$
\alpha\left[\sum_{h=1}^{\infty} D^{h} e_{j}\right] \leqslant \frac{f_{j}}{1-k} \gamma .
$$

Similarly, for some specified row vector with $k<1$,

$$
\alpha\left[\sum_{h=1}^{\infty} e_{i}^{\prime} D^{h}\right] \leqslant \frac{f_{i}}{1-k} \gamma^{\prime} .
$$

4. Error Bounds on the Approximate Solution. The application of (6) and (7) to stating error bounds on the approximate solution is immediate. It follows from (4) that

$$
\alpha(\epsilon) \leqslant\left[I+\alpha\left(\sum_{h=1}^{\infty} D^{h}\right)\right] \alpha(D x),
$$

and (8) may be coupled with (6) or (7). It is true that (8) requires the matrix multiplication $D x$, but considering that the larger matrix multiplication $G A$ is required anyhow, it appears wise to benefit from the usually sharper error bounds given by ( 8 ) at the expense of some incremental work. In addition, (3) indicates that $D x$ is the first order correction to the approximate solution $x$, and thus $D x$ can also be used to improve $x$.

If $D x$ is not computed, (4) may be used as follows:

$$
\alpha(\epsilon) \leqslant \alpha\left[\sum_{h=1}^{\infty} D^{h}\right] \alpha(x),
$$

and (9) may be coupled with (6) or (7).

Finally, $x$ may be taken to be a column of $G$, the approximate inverse, and thus, error in $G$ may be also investigated. 
5. Specific Example of Error Bounds on the Approximate Solution. As yet, no mention has been made of how $\gamma$ should be chosen once $D$ is given. Generally speaking, the choice would be to minimize $k$, but this indeed is a serious problem. ${ }^{1}$ Without looking for the optimum choice, inspection of $D$ may give some guide. However, a simple choice for $\gamma$ is $e=\sum_{j=1}^{n} e_{j}$. For example, with $D=\left(d_{i j}\right)$, let

$$
\begin{aligned}
k & =\max _{j} \sum_{i}\left|d_{i j}\right|, \\
f_{i} & =\max _{j}\left|d_{i j}\right| .
\end{aligned}
$$

It is then seen that

If $k<1$, (7) gives

$$
\begin{aligned}
e^{\prime} \alpha(D) & \leqslant k e^{\prime}, \\
e_{i}^{\prime} \alpha(D) & \leqslant f_{i} e^{\prime} .
\end{aligned}
$$

whence from (9)

$$
\alpha\left[\sum_{h=1}^{\infty} e_{i}{ }^{\prime} D^{h}\right] \leqslant \frac{f_{i}}{1-k} e^{\prime},
$$

$$
\alpha\left(e_{i}^{\prime} \epsilon\right) \leqslant \frac{f_{i}}{1-k} e^{\prime} \alpha(x) .
$$

Clearly, with the methods presented, many other error bounds such as (10) are possible.

Carbide and Carbon Chemicals Company

$$
\text { A. DE LA GARZA }
$$

K-25 Plant, Oak Ridge, Tennessee

1 The referee makes the following comments:

"If all $\left|d_{i j}\right|>0$, the optimum choice of $\gamma$ is unique and is the eigenvector $u_{1}$ of $\alpha(D)$ whose components are all positive, and $k$ is then the dominant eigenvalue $\lambda_{1}$ of $\alpha(D)$. This follows from a lemma that, since all $g_{i}>0, \lambda_{1}$ lies strictly between the minimum and maximum of the ratios $e_{i}^{\prime} \alpha(D) \gamma / e_{i}^{\prime} \gamma$, unless the ratios are all equal (and hence equal to $\lambda_{1}$ ). The lemma is a slight extension of Theorem I of HAZEL PERFECT, 'On matrices with positive elements,' Quart. Jn. of Math., s. 2, v. 2, 1951, p. 286-290.

"The vector $\alpha^{p}(D) e$, which is asymptotically a multiple of $u_{1}$ as $p \rightarrow \infty$, may be a useful approximation to $\gamma$ for sufficiently large $p . "$

\section{RECENT MATHEMATICAL TABLES}

1065[A].-H. S. UHLER, "Many-figure approximations for $\sqrt[3]{2}, \sqrt[3]{3}, \sqrt[3]{4}$, and $\sqrt[3]{9}$ with $\chi^{2}$ data," Scripta Math., v. 18, 1952, p. 173-176.

Values of the constants mentioned in the title are given to $710,709,478$, and 478 decimals respectively.

The author reports errata in the values of the cube roots of 2 and 3 as given by BOoRMAN. ${ }^{1}$ These are correct only to $198 \mathrm{D}$ and $54 \mathrm{D}$ instead of the $305 \mathrm{D}$ and $241 \mathrm{D}$ given.

There is given also data on the distribution of the digits of each of the four cube roots. Values of $\chi^{2}$ and the associated probabilities are given for the first $50 k$ digits for $k=1,2, \cdots$. No unusual distributions are revealed. D. H. L.

' J. M. Boorman, "Square-root notes," The Mathematical Magazine, v. 1, 1887, p. 208. 